\title{
The AMP-activated protein kinase AAK-2 links energy levels and insulin-like signals to lifespan in C. elegans
}

Javier Apfeld ${ }^{1}$ Greg O'Connor, Tom McDonagh, Peter S. DiStefano, and Rory Curtis

Elixir Pharmaceuticals, Cambridge, Massachusetts 02139, USA

Although limiting energy availability extends lifespan in many organisms, it is not understood how lifespan is coupled to energy levels. We find that the AMP:ATP ratio, a measure of energy levels, increases with age in Caenorhabditis elegans and can be used to predict life expectancy. The $C$. elegans AMP-activated protein kinase $\alpha$ subunit AAK-2 is activated by AMP and functions to extend lifespan. In addition, either an environmental stressor that increases the AMP:ATP ratio or mutations that lower insulin-like signaling extend lifespan in an aak-2-dependent manner. Thus, AAK-2 is a sensor that couples lifespan to information about energy levels and insulin-like signals.

Supplemental material is available at http://www.genesdev.org.

Received August 27, 2004; revised version accepted October 18,2004

Interventions that limit energy availability extend lifespan in an array of organisms (Weindruch and Sohal 1997; Osiewacz 2002; Tissenbaum and Guarente 2002). A common method to limit energy is dietary restriction, which leads to lifespan extension in organisms as diverse as yeasts, nematodes, and rodents (Lakowski and Hekimi 1998; Longo and Finch 2003). However, little is known about how aging is coupled to information about the energy state of the animal.

In humans, aging is accompanied by an increase in mitochondrial dysfunction in muscle (Petersen et al. 2003), which is expected to lower cellular energy levels. Similarly, senescent human fibroblasts have a higher AMP:ATP ratio, a sensitive measure of energy levels, than do young fibroblasts (Hardie and Hawley 2001; Wang et al. 2003). We speculated that animals actively sense changes in energy levels and respond by adjusting their lifespan. To investigate this possibility, we studied the role of an AMP-activated protein kinase (AMPK) in the regulation of lifespan in Caenorhabditis elegans.

AMPK belongs to a conserved family of eukaryotic protein kinases that function as energy sensors to coordinate the response to conditions that lower energy lev-

[Keywords: Aging; C. elegans; AMPK; insulin signaling; FOXO; energy levels]

${ }^{1}$ Corresponding author.

E-MAIL japfeld@elixirpharm.com; FAX (617) 995-7014.

Article published online ahead of print. Article and publication date are at http://www.genesdev.org/cgi/doi/10.1101/gad.1255404. els (Hardie and Hawley 2001). For example, when energy availability is limited in rodents, AMPK functions to restore normal energy levels by stimulating glucose uptake in skeletal muscle and glycolysis in the heart and by promoting feeding by regulating a hypothalamic circuit (Marsin et al. 2000; Mu et al. 2001; Andersson et al. 2004; Minokoshi et al. 2004). AMPK is activated by AMP and inhibited by ATP via an allosteric mechanism. Thus, AMPK is a sensor of low energy levels and becomes active when the AMP:ATP ratio is high. AMPK is a heterotrimeric complex that consists of a catalytic $\alpha$ subunit and regulatory $\beta$ and $\gamma$ subunits. Here, we report that the $C$. elegans AMPK $\alpha$ subunit AAK- 2 is activated by AMP and functions to extend lifespan. We find that a high-temperature pulse (HTP), an environmental stressor that lowers energy levels, extends lifespan and lowers fertility in an aak-2-dependent manner. Insulin-like signaling regulates lifespan in C. elegans, Drosophila, and rodents (Tissenbaum and Guarente 2002); and we find that aak-2 and $d a f-16 / F O X O$ function in parallel to mediate the lifespan extension of daf-2/Insulin-like Receptor mutants. Together, our findings indicate that AAK-2 is a sensor that couples lifespan to information about energy levels and insulin-like signals.

\section{Results and Discussion}

To investigate whether changes in energy levels are part of the normal aging process in C. elegans, we first measured the AMP:ATP ratio as a function of age. The AMP: ATP ratio in living animals increases from $<0.1$ at day 4 of adulthood to 0.8 at day 18 , an age near the maximum lifespan of the population (Fig. 1A; Supplementary Table 2 ). Since the AMP:ATP ratio changes with age, we also asked whether the AMP:ATP ratio is correlated with life expectancy. We recorded the survival of a parallel group of animals and then derived the life expectancy for animals at each age. Linear regression indicated a strong correlation between AMP:ATP ratio and life expectancy (Fig. 1D). Based on this observation, we asked whether differences in AMP:ATP ratio among animals of the same age may serve as a predictor of life expectancy. Aging populations are phenotypically heterogeneous due to stochastic events (Herndon et al. 2002). Among animals of the same age, those that display spontaneous movement (Class I) live longer than those that move only in response to prodding (Class II) (Herndon et al. 2002). We divided living animals into these two classes at days 12 and 14 of adulthood. These groups of animals were then subdivided to measure either the AMP:ATP ratio or lifespan. We found that Class I animals have a lower AMP:ATP ratio than Class II animals and, as expected, live longer (Fig. 1B,C). We used the observed AMP: ATP ratios of Class I and Class II animals to predict life expectancy using the fit previously derived by linear regression (Fig. 1D). The predicted life expectancies of Class I and Class II animals closely match the experimental observations (Fig. 1D; for example, among 12-dold adults, Class I: 4.3 d predicted, $4.1 \mathrm{~d}$ observed; Class II: $1.0 \mathrm{~d}$ predicted, $1.3 \mathrm{~d}$ observed). Therefore, differences in the AMP:ATP ratio reflect the effect of stochastic events on lifespan. It would be interesting to determine if the AMP:ATP ratio correlates with life expectancy in other organisms, including humans. Predictors of life ex- 

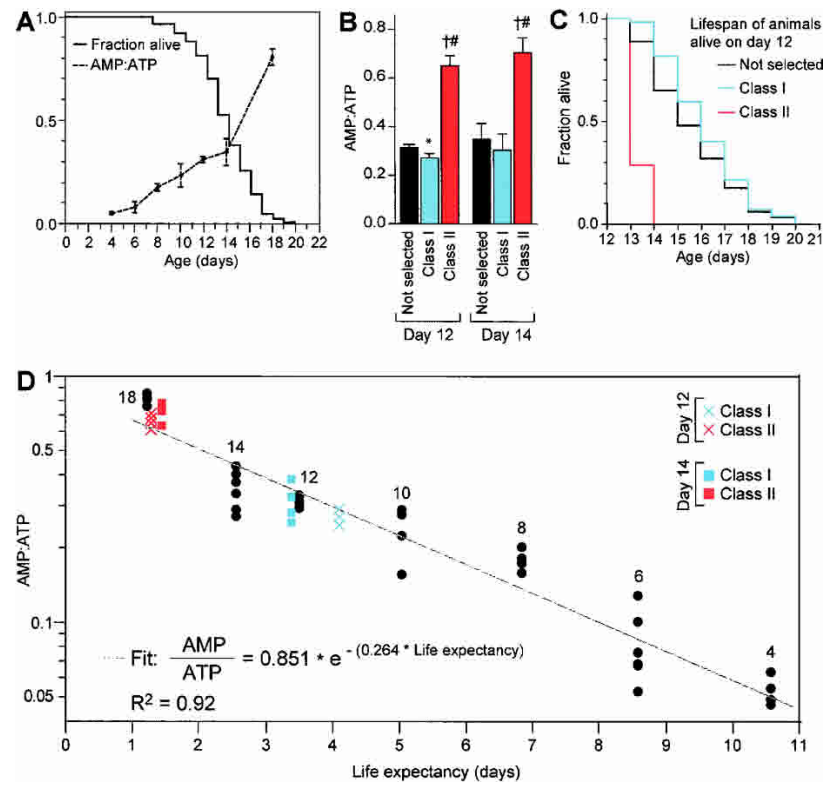

Figure 1. The AMP:ATP ratio predicts life expectancy. (A) Survival curve and increasing AMP:ATP ratio with age. fem-1(hc17); fer15(b26) animals were grown at $25^{\circ} \mathrm{C}$; their lack of sperm at this temperature facilitates growing a large number of animals. In parallel cultures, either the number of surviving animals or adenine nucleotide measurements were recorded at the times indicated. $(B)$ AMP:ATP ratios of 12- and 14-d animals divided based on their motility. "Not selected" represents a group of animals that was not separated based on motility. ${ }^{\star} P<0.01$ and $+P<0.0001$, compared with "not selected"; $\# P<0.0001$ compared with Class I (unpaired $t$-test). (C) Survival curves of 12-d Class I or Class II or "not selected" animals based on motility. Similar data were obtained for 14-d animals. For statistical data on lifespans see Supplementary Table 1. $(D)$ Least squares regression of the AMP:ATP ratio as a function of life expectancy of animals described in A. The age of "not selected" animals is noted next to the data points. The fit and $\mathrm{R}^{2}$ values were determined from "not selected" animals only. Twelve- and fourteen-day Class I and Class II animals were plotted on this graph according to AMP:ATP ratio and life expectancy (measured in $B, C$ ), actual life expectancy closely correlated with values predicted based on AMP:ATP ratio. The increase in the AMP:ATP ratio with age is not due solely to an increase in the fraction of Class II relative to Class I animals, because the AMP:ATP ratio of day-12 Class I animals is higher than that of day-4 animals, which are all Class I $(P<0.0001$, unpaired $t$-test $)$.

pectancy such as the AMP:ATP ratio may be useful clinically as predictors of susceptibility to age-dependent diseases, such as Alzheimer's and cardiovascular disease.

Since energy levels decrease with age and predict life expectancy in C. elegans, a mechanism that senses energy levels may function to regulate lifespan. One possible candidate is the AMPK complex (Hardie and Hawley 2001). We identified two AMPK $\alpha$ subunit homologs in C. elegans, AAK- 1 and AAK-2, which are $52 \%$ and $40 \%$ identical to human AMPK $\alpha 1$, respectively, and are also related to the invertebrate SNF1 proteins (Fig. 2A; Supplementary Fig. 1). The kinase domains of AAK-1 and AAK-2 share $80 \%$ and $71 \%$ amino acid identity, respectively, with the kinase domain of the human AMPK $\alpha 1$ subunit, including conservation of a critical threonine residue whose phosphorylation is required for AMPK activation (Hardie and Hawley 2001). While preliminary evidence suggests that aak-1 does not appear to be required for the control of lifespan (data not shown), aak-2(ok524) mutants have a 12\% shorter lifespan than wild-type animals (Fig. 2B). Moreover, a lipofuscin-like fluorescent pigment that accumulates in an age-dependent manner in the intestine (Garigan et al. 2002; Herndon et al. 2002) accumulates at a faster rate in aak-2 mutants than in wild-type animals (Fig. 2C). aak-2 mutants appear otherwise healthy since they move, feed, and defecate normally (Supplementary Fig. 2). Together, these results suggest that the shortened lifespan of the aak-2 mutant is due to accelerated aging. In addition, transgenic animals with a higher aak-2 gene dose live on average $13 \%$ longer than controls (Fig. 2B). Thus, the aak-2 gene regulates lifespan in a dose-dependent manner.

To determine whether AAK-2 possesses AMPK activity, we asked whether it can phosphorylate a classical AMPK substrate, the SAMS peptide, and whether it is regulated by AMP and phosphorylation (Davies et al. 1989; Stein et al. 2000). An AAK-2-GFP fusion protein immunoprecipitated from C. elegans extracts phosphorylates the SAMS peptide. Moreover, its kinase activity is increased by a factor of three by AMP, with a half-maximal effect at $2.3 \mu \mathrm{M}$ (Fig. 2D). Immunoprecipitates from control GFP transgenic animals do not show SAMS kinase activity (data not shown), indicating that the kinase activity of AAK-2-GFP is due to AAK-2. We also found that treatment of AAK-2-GFP with protein phosphatase-1 reduces the kinase activity by $90 \%$ (Fig. 2E). This indicates that AAK-2 is normally activated by phosphorylation, and that phosphorylation is necessary for AAK-2 activity even in the presence of AMP. Thus, AAK-2 exhibits the hallmarks of an AMPK (Hardie and Hawley 2001).

We also studied the role of $a a k-2$ in the context of other conditions that affect lifespan. In many organisms, lifespan can be extended by a sublethal dose of a stressor early in life (hormesis) (Minois 2000). In C. elegans, treatment early in life with a pulse of high temperature, high oxygen pressure, or oxidative stress extends lifespan (Cypser and Johnson 2002). We asked whether environmental stressors influence the AMP:ATP ratio. Exposure of $C$. elegans to high temperature, starvation, or mitochondrial poisoning causes an increase in the AMP:ATP ratio, which is reversed upon return to normal growth conditions (Fig. 3A). Thus, the AMP:ATP ratio changes in response to the presence of environmental stress. We then determined whether the hormetic effect of high temperature on lifespan requires aak-2 activity. Prefertile 1-h-old wild-type adults exposed to an HTP of $35^{\circ} \mathrm{C}$ for $2 \mathrm{~h}$ live $\sim 30 \%$ longer than untreated controls (Fig. 3B). In contrast, the same treatment does not affect the lifespan of aak-2(ok524) mutants (Fig. 3B). Therefore, aak-2 is required for the lifespan extension caused by high-temperature stress. We propose that the increase in the AMP:ATP ratio that follows high-temperature stress leads to lifespan extension by increasing AAK-2 activity (Fig. 4C). As the lifespan increase outlasts by many days the transient increase in AMP:ATP ratio that occurs early in adulthood, it is likely that the "molecular memory" of the stressor is mediated by AAK-2 phosphorylation of downstream targets.

We reasoned that the inability to sense and respond to stress-induced increases in the AMP:ATP ratio may render aak-2 mutants less able to cope with stress. Indeed, aak-2(ok524) mutants are more sensitive than wild-type animals to killing by either high temperature (Fig. 3C) or mitochondrial poisoning (Fig. 3D). We also measured the 

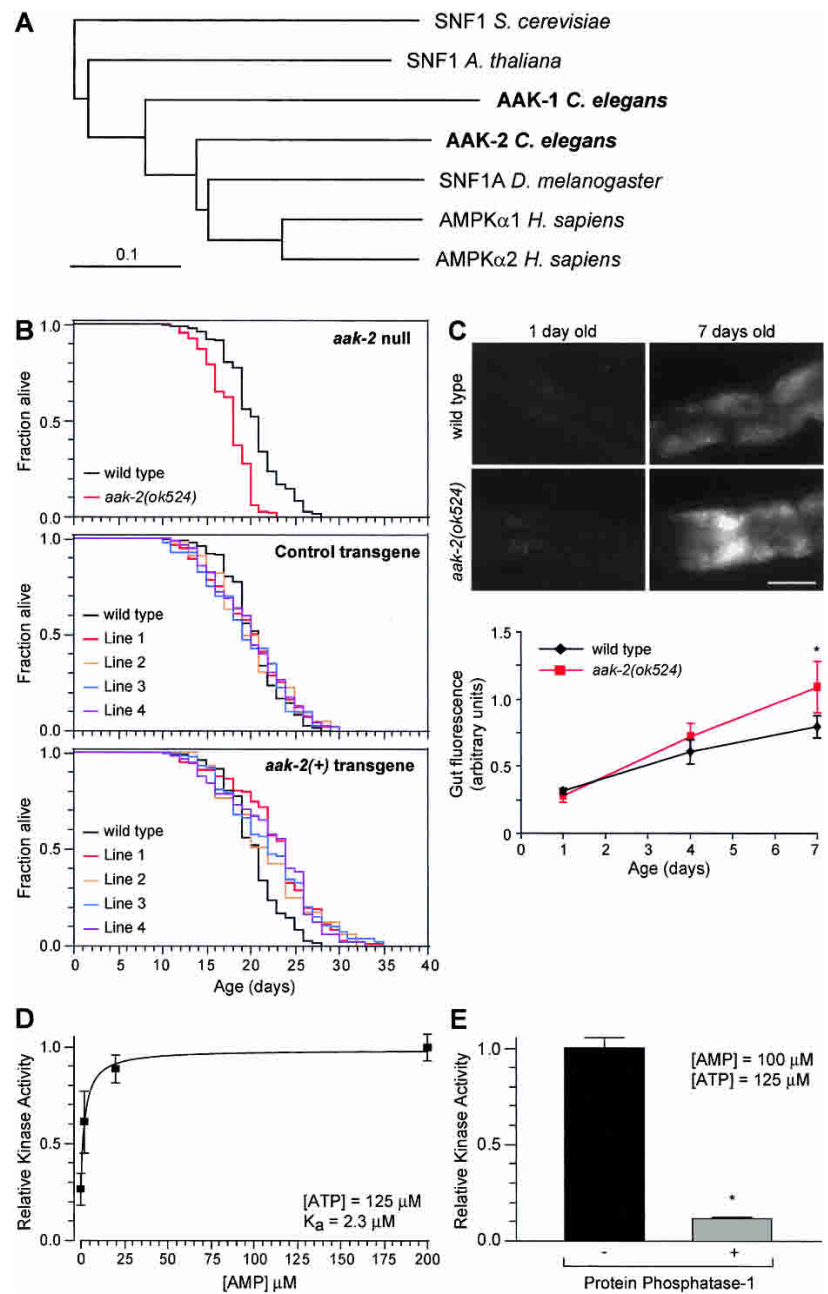

Figure 2. AAK-2 has kinase activity and functions to extend lifespan. (A) Phylogenetic analysis of the AAK-1 and AAK-2 sequences. Additional sequences used were hAMPK $\alpha 1$ (GI:20178277), hAMPK $\alpha 2$ (GI:20178276), DmSNF1A (GI:17137472), AtSNF1 (GI: 30678280), and ScSNF1 (GI:6320685). Bar is 0.1 substitutions per site. (B) Survival curves of animals with decreased, normal, or increased aak-2 gene dosage. For statistical data see Supplementary Table 1. $(C)$ Representative pictures and quantification of fluorescence in the anterior intestine as a function of adult age and genotype (400-msec exposures; bar is $50 \mu \mathrm{m}$; anterior is left, dorsal is up). ${ }^{\star} P<0.0001$; otherwise $P>0.05$ (unpaired $t$-test). $(D, E)$ Measurements of kinase activity in AAK-2-GFP immunoprecipitates using the SAMS peptide assay. $(D)$ Increasing the AMP concentration leads to an increase in kinase activity. $(E)$ Treatment with protein phosphatase-1 greatly reduces kinase activity. ${ }^{\star} P<0.0001 \mathrm{com}$ pared with no treatment (unpaired $t$-test).

effect of high-temperature stress on progeny production. Treatment of prefertile 1-h-old wild-type adults with an HTP causes an $82 \%$ decrease in fertility (Lithgow et al. 1994; Fig. 3E). In contrast, aak-2(ok524) mutants show only a $40 \%$ decrease in fertility under those conditions (Fig. 3E). Therefore, exposure to an HTP results in lifespan extension and a decrease in fertility, and these processes are dependent upon aak-2. These results are consistent with the "disposable soma" theory of aging, which predicts that animals have a machinery that actively executes a trade-off between lifespan and fertility under energy-limiting conditions (Kirkwood et al. 2000).
We propose that AAK-2 is an energy sensor for that machinery, since AAK-2 activity directly responds to changes in the AMP:ATP ratio.

We also asked whether aak-2 plays a role in the insulin-like signaling pathway that regulates lifespan (Tissenbaum and Guarente 2002). Reduction-of-function mutations affecting the insulin-like receptor DAF-2 extend lifespan (Kenyon et al. 1993). We measured the lifespans of aak-2(ok524) mutants and daf-2(m577) mutants, as well as daf-2(m577); aak-2(ok524) double mutants. daf-2(m577); aak-2(ok524) double mutants have lifespans that are indistinguishable from those of aak2(ok524) single mutants (Fig. 4A). Double mutants of aak-2(ok524) and stronger daf-2 reduction-of-function alleles also have shortened lifespans compared with daf-2 single mutants (Supplementary Fig. 3), although they live longer than aak-2(ok524) single mutants. Therefore, aak-2-independent as well as aak-2-dependent mechanisms are required to produce the exceptionally long lifespans of $d a f-2$ mutants.

We also examined the role of aak-2 in the regulation of dauer formation, another process regulated by the insulin-like signaling pathway. At high temperature, juvenile daf-2 mutants enter a state of diapause (called "dauer") instead of growing into fertile adults (Riddle and Albert 1997). Rather than arresting as dauers, the daf-2; aak-2 double mutants grow into fertile adults at $25^{\circ} \mathrm{C}$ (Fig. 4B). These results indicate that aak-2 promotes lifespan extension and dauer formation in insulinlike pathway mutants (Fig. 4C). Because the AMP:ATP ratio in daf-2 mutants is identical to that of wild-type animals (Table 1), the mechanism by which daf-2 mutants extend lifespan must be independent of the AMP: ATP ratio.

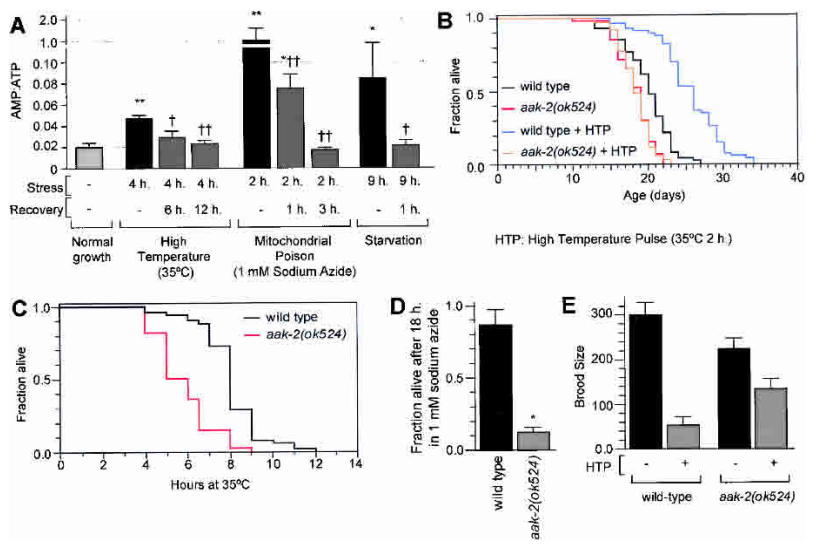

Figure 3. Effect of stress on wild-type and aak-2 mutants. $(A$ AMP:ATP ratio in wild-type animals under normal and stressful conditions. Comparison of stress condition with normal growth $\left({ }^{\star}\right)$, and comparison of stress condition with reversal of stress $(t) .{ }^{*}$ and $\dagger, P<0.01 ;{ }^{*}$ and $+\dagger, P<0.0001$ (ANOVA). (B) Effect of a hightemperature pulse on the lifespans of wild-type animals and aak2(ok524) mutants. (HTP) Pre-fertile 1-h-old adults exposed for $2 \mathrm{~h}$ to $35^{\circ} \mathrm{C}$. For statistical data see Supplementary Table 1. (C) Survival of animals cultured at $35^{\circ} \mathrm{C}$. aak-2(ok524) animals have $28 \%$ shorter lifespans than wild type $(P<0.0001$, logrank test). aak-2(RNAi) animals also have short lifespans at $35^{\circ} \mathrm{C}$ compared with control animals (data not shown). (D) Sensitivity to killing by $1 \mathrm{mM}$ sodium azide for $18 \mathrm{~h} .{ }^{\star} P<0.0001$ compared with wild type (unpaired $t$ test). No untreated control animals of either genotype died after 18 $\mathrm{h}$ (data not shown). (E) Effect of a high-temperature pulse $(2 \mathrm{~h}$ for $35^{\circ} \mathrm{C}$ ) on the brood sizes of wild-type animals and aak-2(ok524) mutants. $P<0.0001$ for all pair-wise comparisons (ANOVA). 

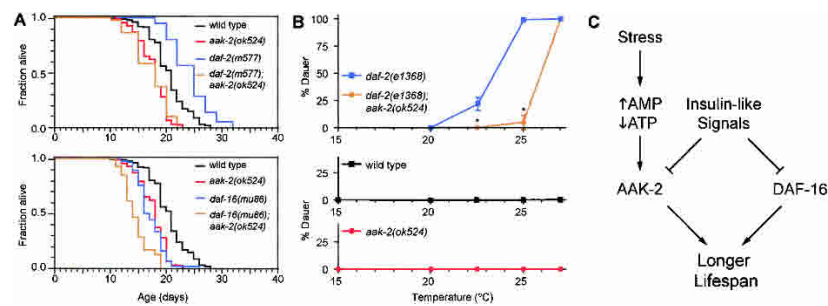

Figure 4. Interactions between $a a k-2$ and insulin-like pathway mutants. (A) Effect of aak-2(ok524) on the lifespans of daf-2 and daf-16 mutants. For statistical data see Supplementary Table 1. (B) Role of aak-2 on dauer formation. ${ }^{\star} P<0.0001$ compared with daf-2(e1368) (ANOVA). Bottom panels show that wild-type animals and aak2(ok524) mutants do not form dauers at the temperatures tested. aak-2(RNAi) also suppresses the dauer-constitutive and lifespan extension phenotypes of daf-2(e1368) mutants (data not shown). (C) Model. AAK-2 regulates lifespan in response to changes in the AMP:ATP ratio and insulin-like signals.

The FOXO transcription factor DAF-16 is required for the lifespan extension of insulin-like pathway mutants (Kenyon et al. 1993; Tissenbaum and Guarente 2002). If aak-2 extends lifespan solely by activating daf-16 or mediating its activity, then absence of aak-2 activity should not further reduce the lifespans of daf-16(mu86) null mutants (Lin et al. 1997). Instead, daf-16(mu86); aak2(ok524) double mutants have 15\% shorter lifespans than either single mutant (Fig. 4A). Therefore, aak-2 is able to influence lifespan in a daf-16-independent manner. These findings indicate that both daf-16 and aak-2 are required for the lifespan extension of $d a f-2$ mutants, and that they act in parallel to influence lifespan (Fig. 4C).

Recent studies have shown that the LKB1 kinase, a tumor suppressor implicated in a broad range of cellular processes, can activate AMPK by direct phosphorylation (Hawley et al. 2003; Woods et al. 2003; Shaw et al. 2004). The C. elegans homolog of $1 k b 1$, par-4, plays a role in the regulation of cell polarity in the early embryo (Watts et al. 2000). We asked whether par-4, like aak-2, also plays a role in the regulation of lifespan and dauer formation. Because par-4 mutants die during early embryogenesis, we used the temperature-sensitive par-4 allele it47 to bypass the embryonic requirement for par- 4 activity. We found par-4(it47ts) partially suppresses the lifespan extension and dauer-constitutive phenotypes of daf2(e1368) mutants (Supplementary Fig. 4). PAR-4 and AAK-2 may function in a common pathway, consistent with PAR-4 activation of AAK-2, since par-4 and aak-2 mutants cause similar post-embryonic phenotypes.

Our findings highlight AAK-2 as a key component of a circuit in $C$. elegans that regulates lifespan in response to environmental stressors and insulin-like signaling (Fig. 4C). This circuit also may be influenced by signals from sensory neurons and the reproductive system, as these inputs modulate the activity of the insulin-like signaling pathway (Apfeld and Kenyon 1999; Hsin and Kenyon 1999). Of note, a similar circuit has been described in the rat heart, where insulin prevents AMPK from being activated by the lower energy levels that occur following ischemia or anoxia (Beauloye et al. 2001). Identification of the targets of AAK-2 phosphorylation will be informative in uncovering the mechanism by which AAK-2 extends lifespan. The regulation of lifespan by this circuit may be evolutionarily conserved since many of its components regulate lifespan in other organisms (Tschape et al. 2002; Tatar et al. 2003). This circuit may have evolved to allow the animal to make an optimal use of its energy resources early in adulthood when reproduction occurs, an idea consistent with the "disposable soma" theory of aging (Kirkwood et al. 2000). We propose that AAK-2 coordinates this process by integrating information from the AMP:ATP ratio and insulin-like signaling. As a result, the lifespan of the animal can be adjusted in response to a wide range of physiological and environmental conditions.

\section{Materials and methods}

General methods and strains

Wild-type C. elegans was Bristol N2. Unless noted, experiments were performed at $20^{\circ} \mathrm{C}$. All experiments involving fem-1(hc17); fer-15(b26) were conducted at $25^{\circ} \mathrm{C}$. The following genes and mutations were used: LG I: daf-16(mu86); LG II: fer-15(b26); LG III: daf-2(e1368), daf-2(e1370), daf-2(m577); LG IV: fem-1(hc17), par-4(it47); LG X: aak-2(ok524).

\section{Gene identification and sequencing}

We identified two C. elegans AMPK $\alpha$ subunits (aak-2/T01C8.1 and aak1/PAR2.3) in WormBase (http://www.wormbase.org, release WS98) by BlastP search with the protein sequences of the human subunits. We confirmed the sequence of $a a k-2$. The ok524 allele of $a a k-2$, generated by the C. elegans Gene Knockout Consortium (http://www.celeganskoconsortium.omrf.org), contains a 409-nucleotide deletion between exon 3 and intron 3, resulting in the insertion of a stop codon. The predicted transcript encodes a protein that truncates after amino acid 164 and, therefore, lacks a complete kinase domain as well as the inhibitory and AMPK $\beta \gamma$-binding domains (Supplementary Fig. 1). Therefore, the ok524 allele is presumed to be a molecular null.

\section{Construction of strains}

Double mutants were constructed by crossing aak-2(ok524) males to the hermaphrodites with the desired second mutation, allowing individual $F_{1}$ progeny to self-fertilize, then identifying $F_{2}$ animals with the phenotype corresponding to the second mutation, transferring these animals to individual plates and identifying aak-2(ok524) homozygotes among their progeny by PCR using primers ATGTCGTTGGAAAGATTCGC and CAATGCTGAGGTGACTTCCTCTTCG. The $27^{\circ} \mathrm{C}$ Daf-c phenotype was used to identify daf-2 mutants. daf-16(mu86) and daf-16(+) were distinguished by PCR (Lin et al. 1997). daf-2(e1368); par-4(it47ts) double mutants were constructed following a similar scheme, starting with par4(it47ts) heterozygous males. par-4(it47) homozygotes were identified as animals whose progeny were all Emb at $25^{\circ} \mathrm{C}$.

Transgenic animals

Transformation rescue was performed using microinjection of $100 \mathrm{ng} / \mathrm{mL}$ PCR-amplified DNA fragments to generate extrachromosomal arrays.

Table 1. Ratios of adenine nucleotides

\begin{tabular}{lccrr}
\hline Genotype & Mean ADP:ATP ratio \pm SD & Mean AMP:ATP ratio \pm SD & $n$ & Energy charge \\
\hline Wild type & $0.069 \pm 0.011$ & $0.020 \pm 0.004$ & 15 & $0.95 \pm 0.01$ \\
daf-2(e1370) & $0.068 \pm 0.009$ & $0.023 \pm 0.002$ & 8 & $0.95 \pm 0.01$ \\
\hline
\end{tabular}

$n$ is the number of independent trials. No difference in ADP:ATP or AMP:ATP ratios was observed among groups $(P>0.1$, unpaired $t$-test). Energy charge is equal to (ATP + 1/2 ADP)/(ATP + ADP + AMP) and represents the extent that adenine nucleotides exist as high-energy phosphates. 
The aak-2 genomic fragment, generated using primers TGGGATTCC GTCAAAGAAGGACATG and AACAGAAACAATCACTCGCTGAA GG, contains $3.0 \mathrm{~kb}$ and $1.2 \mathrm{~kb}$ of DNA upstream and downstream, respectively, of the aak-2 coding sequence. This transgene fully rescues the dauer-defective and lifespan phenotypes of daf-2(e1368); aak2(ok524) double mutants. The cc:: GFP construct (provided by P. Sengupta) expresses GFP in the coelomocytes and was used as a transformation marker. The AAK-2-GFP construct was generated by PCR-fusion of the aak-2 genomic region and a GFP cDNA with an unc-54 3' UTR from pPD95.77, using primers GAAGAGATGAAAAGAGTGGCGGTATG CC, AGGGTCCTCCTGAAAATGTTCCCGAGCCAGTGTTCCAATC AATGC, GCATTGATTGGAACACTGGCTCGGGAACATTTTCAGG AGGACCCT, and AAGGGCCCGTACGGCCGACTAGTAGG as described (Hobert 2002). daf-2(e1368); aak-2(ok524); fyIs2[AAK-2-GFP 100 $\mathrm{ng} / \mathrm{mL}$ ] was generated by integration of the extrachromosomal array by irradiation with ultraviolet light $(254 \mathrm{~nm})$ and out-crossed four times to wild type. fyIs2 fully rescues the Daf-d phenotype of daf-2(e1368); aak2 (ok524) at $25^{\circ} \mathrm{C}$.

\section{Kinase assays}

About 10,000 fyIs2 animals were lysed using a 30-sec pulse from a sonicator in $250 \mu \mathrm{L}$ of IP buffer $(50 \mathrm{mM}$ Tris at $\mathrm{pH} 7.5,50 \mathrm{mM} \mathrm{NaCl}, 50 \mathrm{mM}$ $\mathrm{NaF}, 1 \mathrm{mM}$ EDTA, $1 \mathrm{mM}$ EGTA, $1 \mathrm{mM}$ DTT, $5 \mathrm{mM}$ sodium pyrophosphate, $1 \mathrm{mM}$ PMSF, $10 \mathrm{mM} \beta$ glycerophosphate, $1 \mathrm{mM} \mathrm{NaVO}_{4}$, and a protease inhibitor cocktail [Roche]). Approximately $1 \mathrm{mg}$ of protein extract was incubated with rabbit polyclonal anti-GFP antibody ab290 (Abcam) and immunoprecipitated with protein G Sepharose beads (Amersham) in IP buffer. Immune complexes were collected by brief centrifugation and washed extensively in IP Buffer. AMPK activity in the immunoprecipitates was determined by phosphorylation of the SAMS peptide (HMRSAMSGLHLVKRR) as described (Hardie et al. 2000) using $\left[\gamma^{33} \mathrm{P}\right]$ ATP and counted in a MicroBeta TriLux liquid scintillation counter (PerkinElmer). Autoradiography of the kinase reactions resolved by SDS-PAGE confirmed that the SAMS peptide was phosphorylated. When appropriate, immunoprecipitates were dephosphorylated with 0.5 units of recombinant rabbit protein phosphatase-1 (NEB) for $30 \mathrm{~min}$ at $30^{\circ} \mathrm{C}$ and then washed three times in IP buffer before the SAMS assay.

\section{Lifespan assays}

Lifespan assays were performed as described (Apfeld and Kenyon 1999). At the L4 molt, animals were transferred to plates containing $20 \mu \mathrm{M}$ 5 -fluoro-2'-deoxyuridine (FUDR, Sigma), which kills their progeny as embryos. Control experiments indicated that this concentration of FUDR does not significantly affect lifespan. We used the L4 molt as $t=0$ for lifespan analysis. Life expectancy at each age was calculated as the remaining mean lifespan of the animals that were alive at that age. Assessment of viability and movement was performed as described (Herndon et al. 2002). We used JMP 5.0 (SAS) software to carry out all statistical analysis and to determine means and percentiles.

\section{Nucleotide measurements}

Perchloric acid extracts were obtained by adapting the method reported by Stocchi et al. (1985) to C. elegans. Between 100 and 400 hand-picked live worms were washed with $\mathrm{M} 9$ buffer $\left(22 \mathrm{mM} \mathrm{KH}_{2} \mathrm{PO}_{4}, 34 \mathrm{mM}\right.$ $\mathrm{K}_{2} \mathrm{HPO}_{4}, 86 \mathrm{mM} \mathrm{NaCl}, 1 \mathrm{mM} \mathrm{MgSO}{ }_{4}$ in $\mathrm{H}_{2} \mathrm{O}$ ) and resuspended in $20 \mu \mathrm{L}$ of M9 buffer. Forty microliters of ice-cold $8 \%(\mathrm{v} / \mathrm{v}) \mathrm{HClO}_{4}$ was added and immediately followed by three intervals of $30 \mathrm{sec}$ of sonication and 30 sec on ice. The solution was neutralized with $1 \mathrm{~N} \mathrm{KHCO}_{3}$ and centrifuged briefly, and the supernatant was passed through a $0.2-\mu \mathrm{m}$ filter (Nanosep), and subjected to reversed phase chromatography using a Targa C18 $250 \times 4.6 \mathrm{~mm} \mathrm{5- \mu m} \mathrm{column} \mathrm{as} \mathrm{described} \mathrm{(Stocchi} \mathrm{et} \mathrm{al.} \mathrm{1985).}$ Nucleotides were detected at $260 \mathrm{~nm}$ with a Waters 486 tunable detector. Peak areas were measured using Peak Explorer software. Nucleotide identities were confirmed by comigration with known standards. Unless noted, animals were 1-d-old adults.

\section{Other assays}

Dauer assays, high-temperature treatment, measurements of brood size, and behavioral assays were performed as described (Lithgow et al. 1994; Apfeld and Kenyon 1999; Feng et al. 2001). Starvation was performed by transfer to bacteria-free, peptone-free plates. Treatment with $1 \mathrm{mM}$ sodium azide was performed by transfer to plates with the compound. Gut fluorescence was photographed on the focal plane of the lumen with a
DM505 filter on a Nikon E800 microscope. Average intensity was determined with Metamorph 6.1rl software.

\section{Acknowledgments}

We thank Piali Sengupta for providing the cc:: GFP construct; Mary Sym, Douglas Crawford, Andrew Dillin, Natasha Libina, and Joy Alcedo for protocols; Jennifer Whangbo, Cynthia Kenyon, Lenny Guarente, and members of the Kenyon Lab and Elixir for helpful discussions and comments on the manuscript; and the Elixir community for support and encouragement. Some of the C. elegans strains used in this work were provided by the Caenorhabditis Genetics Center.

\section{References}

Andersson, U., Filipsson, K., Abbott, C.R., Woods, A., Smith, K., Bloom, S.R., Carling, D., and Small, C.J. 2004. AMP-activated protein kinase plays a role in the control of food intake. J. Biol. Chem. 279: 1200512008.

Apfeld, J. and Kenyon, C. 1999. Regulation of lifespan by sensory perception in Caenorhabditis elegans. Nature 402: 804-809.

Beauloye, C., Marsin, A.S., Bertrand, L., Krause, U., Hardie, D.G., Vanoverschelde, J.L., and Hue, L. 2001. Insulin antagonizes AMPactivated protein kinase activation by ischemia or anoxia in rat hearts, without affecting total adenine nucleotides. FEBS Lett. 505: 348-352.

Cypser, J.R. and Johnson, T.E. 2002. Multiple stressors in Caenorhabditis elegans induce stress hormesis and extended longevity. J. Gerontol. A Biol. Sci. Med. Sci. 57: B109-B114.

Davies, S.P., Carling, D., and Hardie, D.G. 1989. Tissue distribution of the AMP-activated protein kinase, and lack of activation by cyclicAMP-dependent protein kinase, studied using a specific and sensitive peptide assay. Eur. J. Biochem. 186: 123-128.

Feng, J., Bussiere, F., and Hekimi, S. 2001. Mitochondrial electron transport is a key determinant of life span in Caenorhabditis elegans. Dev. Cell 1: 633-644.

Garigan, D., Hsu, A.L., Fraser, A.G., Kamath, R.S., Ahringer, J., and Kenyon, C. 2002. Genetic analysis of tissue aging in Caenorhabditis elegans: A role for heat-shock factor and bacterial proliferation. Genetics 161: 1101-1112.

Hardie, D.G. and Hawley, S.A. 2001. AMP-activated protein kinase: The energy charge hypothesis revisited. Bioessays 23: 1112-1119.

Hardie, D.G., Salt, I.P., and Davies, S.P. 2000. Analysis of the role of the AMP-activated protein kinase in the response to cellular stress. Methods Mol. Biol. 99: 63-74.

Hawley, S.A., Boudeau, J., Reid, J.L., Mustard, K.J., Udd, L., Makela, T.P., Alessi, D.R., and Hardie, D.G. 2003. Complexes between the LKB1 tumor suppressor, STRAD $\alpha / \beta$ and $\mathrm{MO} 25 \alpha / \beta$ are upstream kinases in the AMP-activated protein kinase cascade. J. Biol. 2: 28.

Herndon, L.A., Schmeissner, P.J., Dudaronek, J.M., Brown, P.A., Listner, K.M., Sakano, Y., Paupard, M.C., Hall, D.H., and Driscoll, M. 2002. Stochastic and genetic factors influence tissue-specific decline in ageing C. elegans. Nature 419: 808-814.

Hobert, O. 2002. PCR fusion-based approach to create reporter gene constructs for expression analysis in transgenic C. elegans. Biotechniques 32: $728-730$.

Hsin, H. and Kenyon, C. 1999. Signals from the reproductive system regulate the lifespan of C. elegans. Nature 399: 362-366.

Kenyon, C., Chang, J., Gensch, E., Rudner, A., and Tabtiang, R. 1993. A C. elegans mutant that lives twice as long as wild type. Nature $\mathbf{3 6 6}$ : 461-464.

Kirkwood, T.L., Kapahi, P., and Shanley, D.P. 2000. Evolution, stress, and longevity. I. Anat. 197 (Pt 4): 587-590.

Lakowski, B. and Hekimi, S. 1998. The genetics of caloric restriction in Caenorhabditis elegans. Proc. Nat1. Acad. Sci. 95: 13091-13096.

Lin, K., Dorman, J.B., Rodan, A., and Kenyon, C. 1997. daf-16: An HNF3/forkhead family member that can function to double the life-span of Caenorhabditis elegans. Science 278: 1319-1322.

Lithgow, G.J., White, T.M., Hinerfeld, D.A., and Johnson, T.E. 1994 Thermotolerance of a long-lived mutant of Caenorhabditis elegans. I. Gerontol. 49: B270-B276.

Longo, V.D. and Finch, C.E. 2003. Evolutionary medicine: From dwarf model systems to healthy centenarians? Science 299: 1342-1346. 
Marsin, A.S., Bertrand, L., Rider, M.H., Deprez, J., Beauloye, C., Vincent, M.F., Van den Berghe, G., Carling, D., and Hue, L. 2000. Phosphorylation and activation of heart PFK-2 by AMPK has a role in the stimulation of glycolysis during ischaemia. Curr. Biol. 10: 1247-1255.

Minois, N. 2000. Longevity and aging: Beneficial effects of exposure to mild stress. Biogerontology 1: 15-29.

Minokoshi, Y., Alquier, T., Furukawa, N., Kim, Y.B., Lee, A., Xue, B., $\mathrm{Mu}$, J., Foufelle, F., Ferre, P., Birnbaum, M.J., et al. 2004. AMP-kinase regulates food intake by responding to hormonal and nutrient signals in the hypothalamus. Nature 428: 569-574.

Mu, J., Brozinick Jr., J.T., Valladares, O., Bucan, M., and Birnbaum, M.J. 2001. A role for AMP-activated protein kinase in contraction- and hypoxia-regulated glucose transport in skeletal muscle. Mol. Cell 7: 1085-1094.

Osiewacz, H.D. 2002. Genes, mitochondria and aging in filamentous fungi. Ageing Res. Rev. 1: 425-442.

Petersen, K.F., Befroy, D., Dufour, S., Dziura, J., Ariyan, C., Rothman, D.L., DiPietro, L., Cline, G.W., and Shulman, G.I. 2003. Mitochondrial dysfunction in the elderly: Possible role in insulin resistance. Science 300: 1140-1142.

Riddle, D.L. and Albert, P.S. 1997. Genetic and environmental regulation of dauer larva development. In C. elegans II, pp. 739-768. (eds. D.L. Riddle et al.) Cold Spring Harbor Laboratory Press, Cold Spring Harbor, NY.

Shaw, R.J., Kosmatka, M., Bardeesy, N., Hurley, R.L., Witters, L.A., DePinho, R.A., and Cantley, L.C. 2004. The tumor suppressor LKB1 kinase directly activates AMP-activated kinase and regulates apoptosis in response to energy stress. Proc. Nat1. Acad. Sci. 101: 33293335.

Stein, S.C., Woods, A., Jones, N.A., Davison, M.D., and Carling, D. 2000. The regulation of AMP-activated protein kinase by phosphorylation. Biochem. J. 345 (Pt 3): 437-443.

Stocchi, V., Cucchiarini, L., Magnani, M., Chiarantini, L., Palma, P., and Crescentini, G. 1985. Simultaneous extraction and reverse-phase high-performance liquid chromatographic determination of adenine and pyridine nucleotides in human red blood cells. Anal. Biochem. 146: $118-124$.

Tatar, M., Bartke, A., and Antebi, A. 2003. The endocrine regulation of aging by insulin-like signals. Science 299: 1346-1351.

Tissenbaum, H.A. and Guarente, L. 2002. Model organisms as a guide to mammalian aging. Dev. Cell 2: 9-19.

Tschape, J.A., Hammerschmied, C., Muhlig-Versen, M., Athenstaedt, K., Daum, G., and Kretzschmar, D. 2002. The neurodegeneration mutant lochrig interferes with cholesterol homeostasis and Appl processing. EMBO I. 21: 6367-6376.

Wang, W., Yang, X., Lopez de Silanes, I., Carling, D., and Gorospe, M. 2003. Increased AMP:ATP ratio and AMP-activated protein kinase activity during cellular senescence linked to reduced HuR function. J. Biol. Chem. 278: 27016-28023.

Watts, J.L., Morton, D.G., Bestman, J., and Kemphues, K.J. 2000. The C. elegans par-4 gene encodes a putative serine-threonine kinase required for establishing embryonic asymmetry. Development 127: $1467-1475$.

Weindruch, R. and Sohal, R.S. 1997. Seminars in medicine of the Beth Israel Deaconess Medical Center. Caloric intake and aging. N. Engl. J. Med. 337: 986-994.

Woods, A., Johnstone, S.R., Dickerson, K., Leiper, F.C., Fryer, L.G., Neumann, D., Schlattner, U., Wallimann, T., Carlson, M., and Carling, D. 2003. LKB1 is the upstream kinase in the AMP-activated protein kinase cascade. Curr. Biol. 13: 2004-2008. 